\title{
Book Review \\ Evaluating Sustainable Development in the Built Environment, 2nd Edition
}

Peter S Brandon, Patrizia Lombardi, Wiley-Blackwell Publishing, 2011, 280 pages, ISBN: 978-1-4051-9258-3 (Hardcover), AUD 125.00 NZD 140.00, USD 96.99, GBP 60.95, EURO 79.90

The title of this book being "Evaluating Sustainable Development in the Built Environment" is ideally suited as the content is directly aligned with the keywords of built environment, development and sustainability. The authors (Peter S. Brandon and Patrizia Lombardi) have produced a comprehensive text which addresses one of the major challenges in the built environment, namely how to ensure stakeholders fully understand and implement the rapidly changing area of sustainable development. This second edition has been completely rewritten and updated from the successful first edition, as well as including many new and emerging insights into this field.

The book is supported by detailed testimonials from highly regarded academics in different European universities, indicating to the reader this text is worthy of respect before even opening the cover. Evaluating Sustainable Development in the Built Environment covers 264 pages in approximately A5 page size and is divided into nine chapters. The chapters have been carefully organised into a sequential order which commences with a broad scene setting introduction and then progresses to the complex challenges with sustainable development. At the end of the text is a comprehensive index and is accompanied with a list of relevant websites.

The book commences with an informative overview of the two authors and their respective backgrounds, following by a preface by the authors and a single page of acknowledgements. The nine chapters are divided into a smaller number of sub-headings with a reference list and also a bibliography inserted after the last chapter. Most chapters also include a summary section which is very helpful to the reader to highlight the pertinent aspects in each chapter. Chapter one (Setting the Context for Evaluating Sustainable Development) introduces the environmental perspective and the varying perceptions about sustainability. The discussion moves to the international policy debate and the impact of sustainability on the built environment, prior to seeking to define what sustainability actually is and discussing management and intervention considerations for sustainable development.

Chapter two (Time and Sustainability) examines the complex background to sustainability changing over time and investigates innovation and stability. This chapter investigates the impact of time on sustainable development including the development lifecycle and time in evaluation aspects. There is an extensive review of relevant literature and useful supporting graphs and diagrams are included.

Following on, chapter three (Approaches to Evaluation) is concerned with different approaches to addressing sustainability in the built environment. The first part refers to the concept of 'The Natural Step' which precedes a discussion about the concept of community capital. This then moves onto the notion of an ecological footprint and a range of alternative evaluation approaches including reference to accounting frameworks. The summary and conclusions section is comprehensive and neatly encapsulates this chapter's intent.

Chapter four (Indicators and Measures) identifies and examines indicators for measuring progress towards sustainable development. It commences by examining the questions behind why it is critical to evaluate and the differences between traditional and sustainable 
development indicators. Reference is made to the type of questions required, namely general or specific, and which international indicators are relevant. The chapter concludes with an informative discussion section following by a succinct summary.

Chapter five (Assessment Methods) investigates an incredibly delicate area with regards to sustainability in the built environment, namely why assessment is undertaken and what is the optimal approach. A comprehensive table of assessment methods, tools and procedures is included as well as a summary of the main assessment methods, tools and procedures in use. This includes a discussion about environmental impact analysis, strategic environmental assessment, cost-benefit analysis, contingent valuation method, hedonic pricing method and other related approaches. The strengths and weaknesses of each approach are discussed and a summary and conclusion section neatly brings the chapter to a close.

Chapter six (A Proposed Framework for Evaluating Sustainable Development) builds on previous five chapters and argues for an optimal framework and the relevant theoretical underpinning that is required. Considerable attention is placed on identifying 15 modalities for understanding sustainable development in the built environment accompanied by a discussion about each. Also included is a checklist of questions segregated for each modality followed by a section which is aimed at synthesising the results. A summary concludes the chapter.

Chapter seven (The Framework as a Structuring Tool: Case Studies) seeks to explain the approach to a multi-modal framework for decision-making by using a series of case studies. Each of the four case studies are evaluated in detail and are based on a municipal waste treatment system, a redevelopment of an urban area, a multi-stakeholder urban regeneration project and a strategic plan for a designated city. Attention is also placed on the key issues of social reporting and followed by a conclusion and summary section.

Chapter eight (Towards Management Systems and Protocols) begins with a broad discussion about management and the appropriate definition thereof. The argument then moves onto the question of who is a manager and what exactly is the concept of the planning framework. Challenges with the management process are examined including possible approaches to management. Attention is then placed on 'The Vancouver Study' which examines the concept of a sustainable city in 100 years.

The final chapter in the book is chapter nine (Education and Research) which is designed to examine the largest single challenge facing sustainable development in the built environment, namely how to educate the stakeholders and also undertake meaningful research. The majority of this chapter is written in a general discussion format and focuses on education, which is then accompanied by a section highlighting the importance of having a research agenda.

Throughout the book there is optimal use of detailed tables, graphs and conceptual diagrams which are referred to frequently throughout the text. Each chapter is well written and the authors have successfully produced a book which provides a fluent chapter-by-chapter insight into this changing topic. The book is extremely well organised and set out, which in turn assists the reader to gain an insight into this increasingly important area. The typical reader's knowledge about sustainability can range from very limited to a relatively expert, since the text is well written and caters for a wide range of stakeholders. In conclusion the text is recommended as direct and informative insight into the otherwise complex area of sustainable development in the built environment.

Professor Richard Reed

Deakin University, Australia

Reed, R (2012) Book review, Australasian Journal of Construction Economics and Building, 12 (1) $93-94$ 